\title{
The World of Framsticks: Simulation, Evolution, Interaction ${ }^{1}$
}

\author{
Maciej Komosinski \\ Institute of Computing Science, \\ Poznan University of Technology \\ Piotrowo 3A, 60-965 Poznan, Poland \\ maciej.komosinski@cs.put.poznan.pl \\ http://www.frams.alife.pl/
}

\begin{abstract}
A three-dimensional virtual world simulation is described, where evolution takes place and it is possible to investigate behaviors of creatures in real-time. Bodies of these creatures are made of sticks, and their brains are built from artificial neurons. There are no constraints on topology and complexity of neural networks, as well as on the size of morphology. The model is inspired by biology, so energetic issues such as energy gains and losses are also considered. The evolutionary process can be guided by some pre-defined criteria, however, it is possible to mimic spontaneous evolution when the fitness is defined as the life span of the organisms. Interactions in the virtual world are discussed (including the possibility of worldwide-distributed simulation), and the results of so-far experiments are presented.
\end{abstract}

\section{Introduction}

Systems qualifying as virtual worlds have been built for a long time, but it is only recently that such systems achieved a superior level of realism, opening up the way for new applications. This is mainly due to the rapid development of computing machines, which now allow for fast simulation of complex systems. Such systems could earlier be investigated only theoretically. Another feature of present virtual world simulations is the existence of possible areas of their application, which range from medicine and economy to entertainment.

Creating artificial worlds usually involves simulation of an environment and its habitants, interacting with each other and with the world they live in. Whereas such simulations of complex worlds are popular, it is not so easy to find the property of nature-like evolution included in the model $[8,14,18]$. Yet the evolution is an important source of complexity (emergence), because the amount of complexity explicitly designed in a system via its rules is limited. In particular, evolution is a force that can produce complexity going beyond the reach of the initial design. There

\footnotetext{
${ }^{1}$ Published in: Proceedings of $2^{\text {nd }}$ International Conference on Virtual Worlds, Paris, France,
} July 2000. (C) Springer-Verlag (LNAI 1834), pp. 214-224. 
are many studies that focus on evolution of living forms within the field of Artificial Life [12]. However, these simulations are usually kept simple to focus on underlying mechanisms and allow for their efficient analysis and study $[4,5,6]$.

The system described here tries to comprise the typical features of virtual world, together with its attractiveness for the human user, and to allow for evolution of the creatures which are simulated within the world. Although the simulation captures currently only a subset of properties of the natural world, its assumptions are inspired by natural laws $[2,17]$. Moreover, the properties included are rich enough to allow a host of interactions to develop. Such a setup guarantees that the whole system is attractive for its observer/participant, and the evolutionary adaptation property together with the realistic three-dimensional simulation seem to be its most important features.

While there have been experiments with the guided evolutionary processes in sophisticated systems [20], there was no attempt at spontaneous evolution using environments and creatures as complex as Framsticks. We hope that the presented model is suitable to give rise to sophisticated, real-like dependencies and behaviors. If they appear as the result of the spontaneous evolution, they will most probably astonish the beholder of Framsticks' virtual world, thus displaying the emergence property [19].

In Framsticks, the three-dimensional world and creatures (their "bodies" and "brains") are simulated. The creatures are capable of interacting with themselves (locating, pushing, hurting, killing, eating, etc.) and the environment (walking, swimming, ingesting, etc.). Framsticks are made of "sticks", which allows for fast simulation, thus letting the evolution occur in a reasonable time. The virtual world itself can be composed of any combination of flat land, hills, and water. Despite simple simulation, this setup allows for realistic behaviors.

Evolutionary process may be directed by some pre-defined criteria. One of them is life span, which can be used to mimic spontaneous evolution: the life span of a creature depends directly on energetic issues, and longer life span results in greater reproduction abilities. Various genetic representations are proposed, each of which is based on different qualitative ways of creature development.

The paper is organized as follows: section 2 describes Framsticks system, its architecture, and models of simulation and evolution. Section 3 is devoted to the evolutionary properties of the system, describing various genotype representations, genetic operators, etc. Section 4 presents abilities of interaction within and with Framsticks simulator, and sections 5 and 6 discuss results of experiments, summarize the work and present future goals of the project.

\section{Simulation}

\subsection{System architecture}

In general, our main goal is to design the model so that it allows for spontaneous (with natural selection), open-ended evolution. The creatures live in a threedimensional world, and are controlled by neural networks. Currently, the system is 
more appropriate for guided evolutionary processes: the rules of selection and reproduction are pre-defined in the system architecture. That differs Framsticks from some other artificial life simulators (like Tierra [16] and Avida [1]), however, attempts have been made to avoid explicit reproduction rules [10].

In Framsticks system, the basic evolutionary framework is defined in a similar way, as in evolutionary models designed for optimization, such as genetic algorithms [7, 15] and most of artificial life simulations [3, 20, 21]. Therefore, evolution means optimization of creatures according to some predefined criteria. However, it is possible to mimic spontaneous evolution with the 'directed' model of evolution, when the fitness criterion is directly connected with the survival and reproduction abilities.

The life span criterion is suitable for such a purpose. Using life span as fitness value affects selection phase: the longer a creature lives, the better it reproduces. One could simulate breeding of offspring or laying eggs, the amount of which would be proportional to the life span of the parent individuals, and would depend on their energy. However, there is no need to simulate those specific mechanisms as long as life span directly affects the reproductive success. Such an imitation of spontaneous evolution is reasonable.

Simulating creatures in the virtual world is needed to estimate their fitness. In our model, it is possible to adjust the number $n$ of individuals which are simulated at the same time. Thus, the virtual world does not contain the whole set of individuals, but a userdefined part: the artificial world is a reduced model of the whole ecosystem. The advantage of such an architecture is that a few individuals can be simulated much faster than a few thousand, so one can see the simulation in real-time, study the behavior of creatures, and affect them. Also, there is no need to save the detailed state of all the habitants of the world when the simulation is to be finished (that would mean a large amount of data). Only the genotypes and their multi-criteria performances are

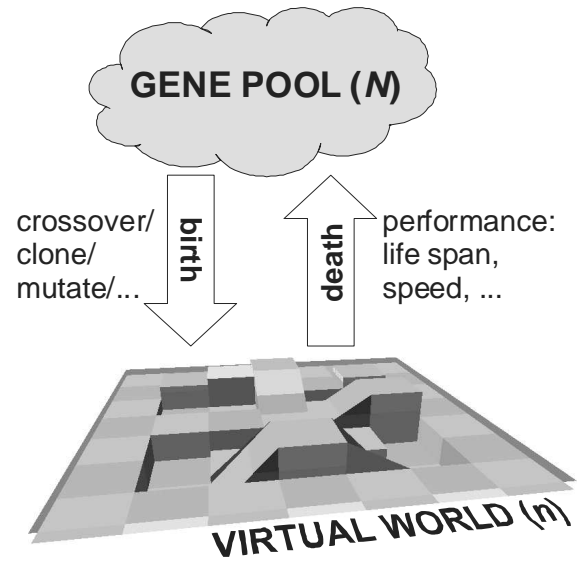

Fig. 1. Architecture of the system. saved.

In order to construct such an architecture (see fig. 1), two parameters are needed: the maximum total number of genotypes, $N$, and the maximum number of individuals simultaneously simulated, $n$. Individuals are selected to be simulated and evaluated when there is a free place in the virtual world (when the number of simulated creatures is less than $n$ ). When an individual dies, the performance of its genotype is updated.

Usually, $n$ is significantly smaller than $N$. When the interaction between simulated creatures is not important, $n$ may be set to 1 (only one organism simulated at a time). 
Larger values of $n$ mean that a larger part of the whole set of individuals is simulated, and more interactions between creatures may happen. Thus, when setting the value for $n$, the size of the virtual world and the goal of evolution should also be considered.

These rules can also be employed when the simulation is distributed. In such a case, it is assumed that the virtual world is not divided into areas or individuals, which would be then simulated in parallel. What is divided is the main gene pool, which is sent in parts to computing sites for evolution, and after some time these sites send their gene pools back to the main process.

\subsection{Physical simulation}

Framsticks simulates a three-dimensional world and creatures (finite element method is used for step-by-step simulation). We decided to use such a sophisticated environment for evolution hoping that a range of complex, various stimuli affecting organisms will be the origin of dynamic development and interesting behaviors will emerge. The environment is used to evaluate individuals (genotypes), and the first behaviors tested were the mechanisms of locomotion and orientation. We considered all the kinds of interaction between physical objects: static and dynamic friction, damping, action and reaction forces, energy losses after deformations, gravitation, and uplift pressure - buoyancy (in water environment). The most important forces are shown on figure 2.

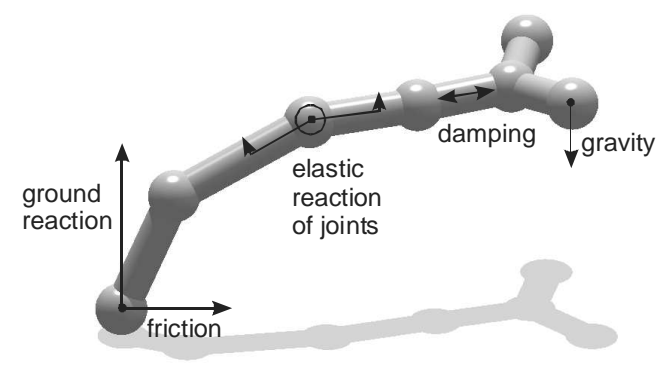

Fig. 2. Forces involved in the simulation.

One should note that there is always a tradeoff between simulation accuracy and simulation time. We need fast simulation to perform evolution, on the other hand, the system should be as realistic as possible to produce realistic behaviors.

Currently, in order to make the simulation faster and due to the computational complexity, some aspects were discarded: collisions between parts of an organism itself and the movement of a water medium were both ignored. Including these in the simulation would make it more realistic, but would not introduce any qualitatively new (important from the viewpoint of the simulated creatures) phenomena. From the viewpoint of evolution and the investigator of the evolved behaviors, meaningful interactions are more important than very realistic, but too slow simulation [13]. 
The current model implies some constraints concerning structure of organisms. The basic element is a stick made of two flexibly joined particles (finite element method is used for simulation). Sticks have specific properties: biological (muscle strength, stamina, energetic: assimilation, ingestion, and initial energy level), physical (length, weight, friction), and concerning stick joints (rotation, twist, curvedness).

Neurons (connected in any way) and receptors can also be placed on sticks. Rather than evolving neural networks exclusively [3], morphology - "body" is evolved together with "brain" in Framsticks.

Muscles are placed on stick joints. There are two kinds of muscles: bending and rotating. When a muscle is sent a zero signal, it becomes neutral (as if it was absent). Positive and negative changes make the sticks move in either direction - it is analogous to natural systems of muscles, with flexors and extensors. The strength of a muscle determines its effective ability of movement and speed (acceleration). However, stronger muscle consumes more energy during its work. Additionally, an increase in the "strength" property of a muscle results in a decrease of the other properties of a stick.

Framsticks have currently three kinds of receptors (senses): those for orientation in space (equilibrium sense, gyroscope), detection of energy/food (non-directional smell) and detection of physical contact (directional touch). Receptors and effectors are shown on figure 3. Details are illustrated at [11].

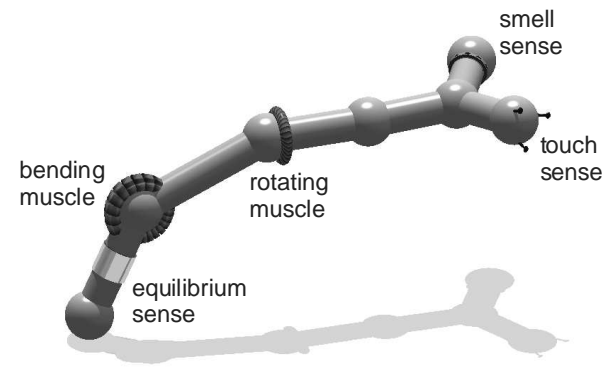

Fig. 3. Senses and effectors of Framsticks.

\subsection{Neural network}

In order to enrich capabilities of creatures' brains, the typical artificial neurons were equipped with three additional parameters which can alter the behavior of each neuron independently. As Nature was our inspiration, we did not introduce sophisticated and unnatural processing units (as in [20]); it is possible to construct complex modules (integrating, differentiating, summing, subtracting, and generators with different shapes) from simple neurons.

The additional neuron parameters (force, inertia and sigmoid) are under control of evolution. They affect the way neurons work: Force and inertia influence changes of the inner neuron state (speed and tendency of changes, respectively). Sigmoid 
coefficient modifies the output function. Details and sample neuronal runs can be found at [11].

The neural network can have any topology and complexity. Neurons can be connected with each other in any way (some may be unconnected). Inputs can be connected to outputs of another neurons, constant values, or senses, while outputs can be connected to inputs of another neurons or effectors (muscles).

\section{Evolution}

\subsection{Genotype representations}

In order to test the properties and abilities of evolutionary processes, various genotype encodings have been designed. All of them are expressed with symbols, so they can be relatively easily investigated, modified and understood. All of them combine "body" and "brain" in the same genotype, so that both morphology (body made of sticks) and control (brain made of neurons) evolve at the same time.

The common basic layer for describing a phenotype is the basic direct encoding. It is least restrictive and allows for building of any creatures which can be simulated within the virtual world. Such a genotype simply lists all the parts of an organism.

The recurrent direct encoding was designed to allow human users to build their own creatures in a possibly simple way, nevertheless some important properties of the encoding were also maintained to make the evolution efficient. Generally, users can write recurrent genotype expressions, which are interpreted as phenotypes. Due to the recurrent nature of the language, the morphology has a tree-like structure. Special signs can be used to modify (increase or decrease) properties of parts of the body (see 2.2). "Brain" is made of neurons with their connections described relatively. Two genetic operators are used: crossover and mutation. It often happens that genotypes are invalid after crossover and mutation because of small errors. That is why a simple repair procedure is used. This procedure can fix little mistakes (like references to nonexisting neurons) and validate an invalid genotype.

In nature, the genetic code of complex organisms does not encode their body layout directly, but rather their process of development. That is why a developmental encoding was designed. An interesting merit of developmental encoding is that it can incorporate symmetry and modularity, features commonly found in natural systems. The developmental encoding is similar to the recurrent direct one, but codes are interpreted as commands by cells (sticks, neurons, etc.). Cells can change their parameters, and divide.

Another encodings (like direct similarity development and implicit embryogeny development) are also proposed in order to test various representations of creatures (morphologies and brains), and the influence of the representations on the evolutionary process. Trying various approaches to describe creatures is also important from the viewpoint of a human user: some representations may be better than others to use in a graphical, user-friendly editor of phenotypes. It should be as easy as possible to build a creature for non-experts, on the other hand, they should be 
able to control details of such design if needed. It is relatively easy to add support for more encodings suggested by developers.

\subsection{Selection and speciation}

When a new individual has to be created, the simulator selects an existing genotype using the standard roulette wheel rule [7], proportionally to its fitness. The genetic operators are then applied.

In nature, groups of similar individuals share the same ecological niche and constitute one species. In Framsticks, similarity to other coexisting species may lower the given species' fitness [7]. This introduces a pressure for populations of species to diversify. The second mechanism which allows speciation is the specific crossover operation: if possible, corresponding parts of genotypes of similar species are exchanged.

The described mechanism of aiding speciation by lowering fitness of similar genotypes is somehow artificial. However, such a phenomenon happens spontaneously in Framsticks when a large number of individuals are simulated at the same time in the virtual world. Then, depending on the behavior, if many individuals exhibit the same method of achieving energy, their situation will become more difficult, and competition within species will arise.

\subsection{Creatures' evaluation}

During the life span of simulated organisms some features are measured, like distance (center of gravity movements), age, average velocity (equal to distance/age) etc. In practice, movements are not measured from the moment of birth, but from the moment when a creature becomes stable. This is because some oscillations take place immediately after creation, causing shifts of the center of gravity. Measuring those movements would be unfair, as they are not caused by the creature itself, and do not depend on a creature's ability to move on its own.

After a creature dies, all the values of various performance criteria are weighted to produce the final fitness value for the given genotype.

\section{Interaction}

Interaction happens on many levels of the Framsticks system architecture. There is interaction between creatures and their world, where senses are the sources of information for organisms, and muscles are their way to influence their own state and their environment.

Potentially, as there is no limitation concerning the complexity of neural network, creatures can become intelligent, and it is unknown to what degree. Framsticks can learn about their orientation, and sense their world (and, potentially, remember it). From the set of three senses, the smell receptor is the most important. It allows to find food, but also to tell food from other creatures. With the proper use of many smell 
sensors, creatures can virtually discriminate between various morphologies of other habitants, sense their movement, their number, etc. The great help of complex brain allows for the unlimited use and exploitation of smell, even for diversification of species, group and social behaviors, and mutual preferences. If the simulator is very complex, it may resemble The Matrix [22] from the viewpoint of the creatures, and they can learn its imperfections in order to utilize them (as it was already observed, but it was evolution what explored simulator faults).

In Framsticks, there is a lot of the interaction with the human role. It is the human who can affect living creatures, revive them (put into artificial world), kill (remove from the world), move in the world with the "robotic arm", feed them (put energy into the world), cause crashes etc. As Framsticks interface may show simulation in realtime, responses to user actions are instantaneous and highly visual. This facilitates the exploration of many aspects of evolutionary process, like fitness criteria, creation of offspring (mutation, crossover, cloning, etc.), population mixing, partial evolution, etc. Through the process of interactive experimentation, users can develop an understanding of some of the fundamentals of evolutionary dynamics. Users can also affect the gene pool by designing their own creatures, editing genotypes, and modifying and improving them with an instant preview of phenotypes.

It is possible to define the virtual world: its size, hills, slopes, water level, and boundaries (like walls, teleport, or no boundaries). Humans can adjust the architecture for the evolutionary process (maximum numbers for genotypes and simulated individuals), turn on or off destructive collisions (whether collisions break creatures), adjust the rate of auto-feeding, adjust energy usage rates for brain and body work, etc.

As the evolutionary process is the source of development in the Framsticks world, the most powerful ability is to understand and adjust the parameters of evolution, like weights for fitness criteria, origin of offspring (mutation, crossover, cloning, etc.), mutation rates, speciation, the ability to exclude some creatures' elements from evolution, etc.

Finally, the virtual world itself affects a human user, who is influenced by what they can see, the way creatures behave: move, seek energy, swim, walk, fight, etc. This kind of interaction is the subtlest one, as it does not concern the apparent action, but it certainly happens. When the simulation is distributed over Internet [11], the collective, worldwide experiments will connect their participants, and enable sharing of ideas, knowledge and experience of the system, analysis of results, and discussions. Distributing evolution of realistic 3D creatures in large scale may change the way people think about evolution, making it more (or less) unusual and ambiguous. Eventually, experiments with open-ended setups may bring unexpected results.

\section{Results}

The majority of our experiments concerned directed evolution, with fitness defined as speed (on the ground or in water). Many walking and swimming species evolved during these runs [11], and we were able to see the evolution of ideas of "how to move" [9]. In one evolutionary run, a limb was doubled while crossing over, and after some further evolution the organism was able to move with two limbs - one for 
pushing back and one for pulling [11]. We also noticed a case when a limb is simultaneously bent and rotated, which was a more effective method of pushing against the ground. In one case a neuron used its saturation to produce delayed signals, which is a kind of a simple "short-term memory". More sophisticated creatures which were evolved could not be easily examined because of their high complexity.

Generally, as long as there is no really good way of locomotion in a population, there is a tendency to try a lot of ideas. The creatures with stronger muscles, better control and many limbs (to achieve stability) survive. Evolution tends to promote strength, and make the pushing/pulling limbs longer and rougher. The really great innovations seem to be discovered once and then sustained throughout the run, until they are replaced by better ideas. We noticed that it was hard to evolve a lowfrequency signal generator, perhaps because it cannot be created by little, step-by-step improvements. Movement in water was easier to achieve than on land, see figure 4 for example of swimming movement (evolutionary redundancy and random character can be observed).

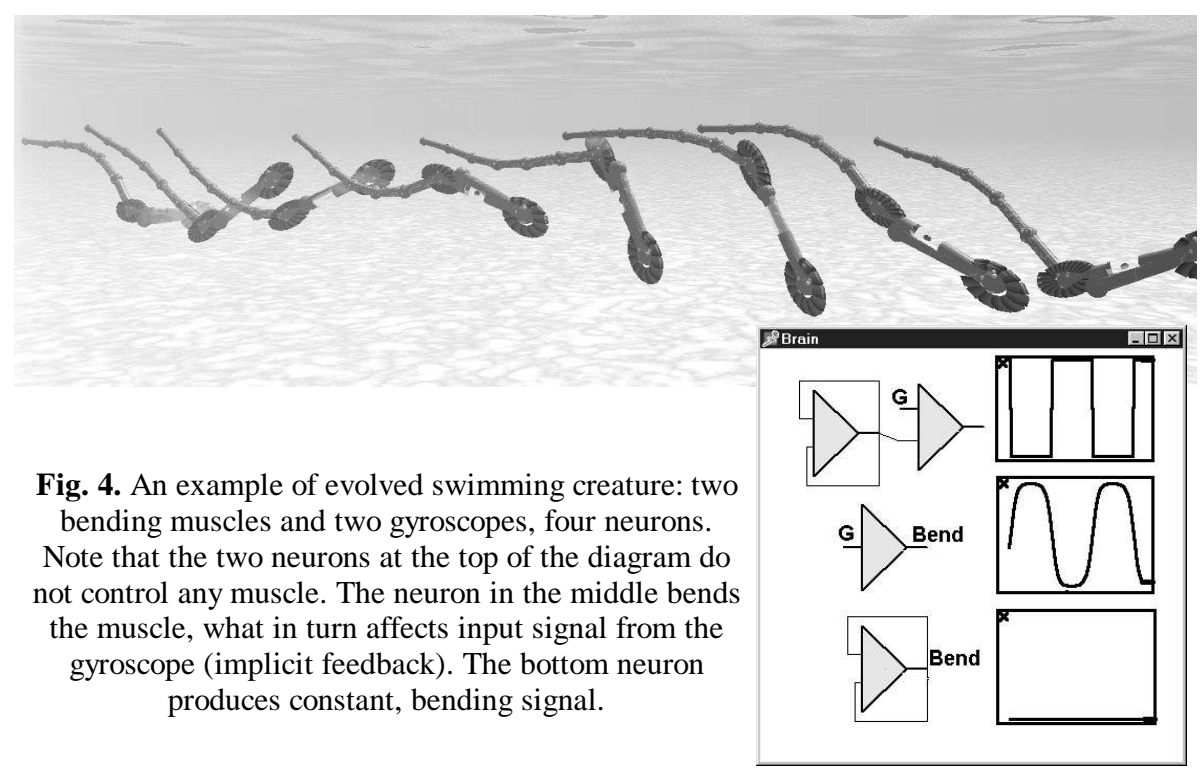

The experiments concerning evolutionary improvement of human pre-designed structures have also been conducted. An example is the evolution of control (neural network) for a pre-designed morphology in order to obtain a creature that lives long. In order to live long, it is required to find energy sources and ingest them. The user can design a genotype, and then turn off crossover and structure mutations, allowing only mutations of neural network (connections, topology and weights). Thus a 
food-seeking creatures are evolved on the basis of pre-designed morphology. See figure 5 for an example of pre-designed body and brain, with further directed evolution of movement.

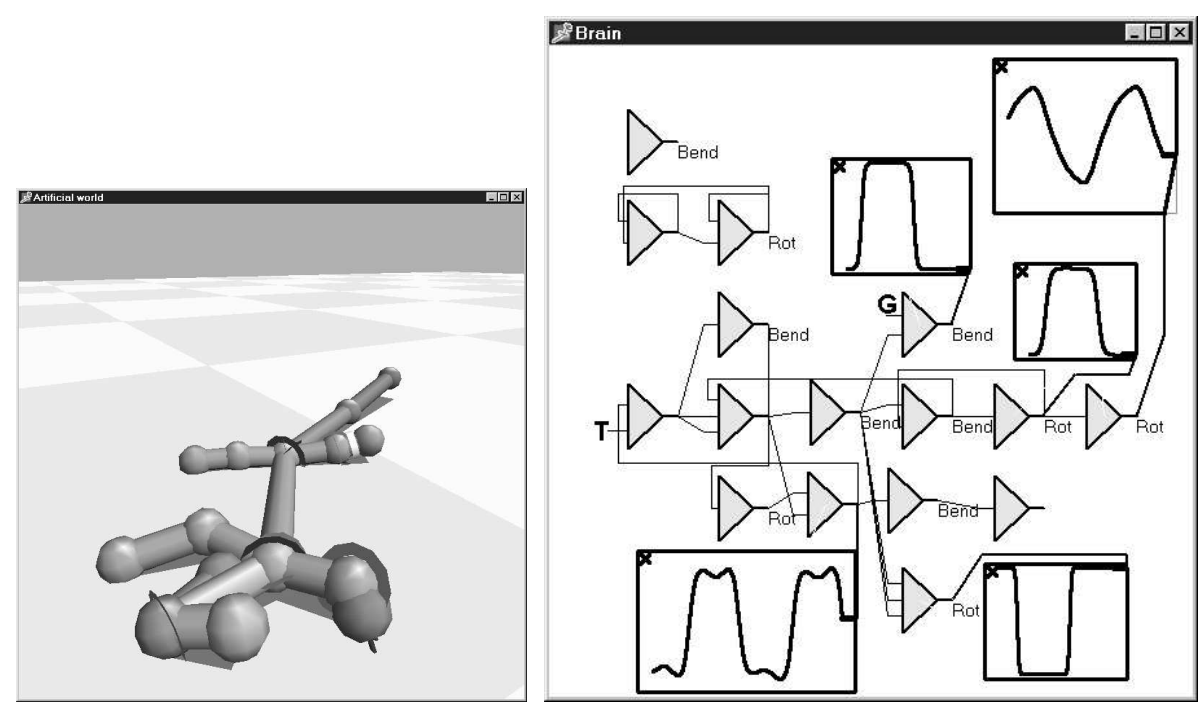

Fig. 5. Pre-designed creature ("lizard"). Neural network connections and weighs evolved with fitness defined as speed. One touch and one equilibrium receptors. Walks in a realistic way.

\section{Discussion and future work}

During our evolutionary experiments, we had to modify simulation rules and fix bugs several times. Evolution turned out to be a very good method of searching the space of solutions (organisms), and was capable of finding good individuals, regardless of their sensibility and validity. Faults in the simulator were sooner or later discovered by evolution and exploited to the highest possible extent in simulated organisms.

There are a few limitations of the current model. The genotypic representations need to be investigated and tuned to allow efficient evolution of creatures, not restricting various, complex body and brain plans. Our future work will thus concern various genotype representations (see 3.1), implementation of specific genetic operators, experiments and their study. We hope that it will make the search of the organism space even more effective. The preliminary results show that it will lead to creation of more diverse constructions and their easier modification. A suitable representation for user-friendly design of creatures will also be investigated.

Our future work will also concern defining a similarity function on the phenotype space, improvements of simulation rules and their parameters, and spontaneous, openended simulations. More receptors and more complex criteria for directed evolutions 
may be introduced. If the increase in computing power permits, we will use more realistic (but slower) physical simulator, so that the evolved creatures will behave more like if they would be built in the real world. Generally, realism and openendedness are properties that require much more computation and effort than simple and/or directed evolutionary models, but we hope to employ Internet worldwide distribution to fully exploit capabilities of the system.

The results we have achieved are promising, and it is really interesting to look forward to the behaviors and phenomena that will emerge with the new genotype encodings, and after collective, distributed experiments are performed.

\section{Acknowledgement}

This work has been supported by the research grant from KBN, Polish State Committee for Scientific Research.

\section{References}

1. Adami, C., Brown, C. T., Evolutionary learning in the 2D artificial life system "Avida". In: Rodney A. Brooks and Pattie Maes (eds.), Artificial Life IV. MIT Press/Bradford Books, 1994.

2. Balkenius, Ch., Natural Intelligence in Artificial Creatures, Ph. D. Thesis, Lund University Cognitive Studies, 1995.

3. Cruse, H., Kindermann, T., Schumm, M., Dean, J., Schmitz, J., Walknet - a biologically inspired network to control six-legged walking. In: Neural Networks 11 (1998) 1435-1447, Elsevier Science Ltd.

4. Dawkins, R., Climbing mount improbable, W.W. Norton \& Company, 1997.

5. Ficici, S. G., Pollack, J. B., Coevolving communicative behavior in a linear pursuer-evader game. In: Proc. Fifth International Conference of the Society for Adaptive Behavior, Pfeifer, Blumberg, Kobayashi (eds.), Cambridge: MIT Press, 1998.

6. Ficici, S. G., Pollack, J. B., Challenges in coevolutionary learning: arms-race dynamics, open-endedness, and mediocre stable states. In: Proc. Sixth International Conference on Artificial Life, Adami, Belew, Kitano, Talor (eds.), Cambridge: MIT Press, 1998.

7. Goldberg, D. E.: Genetic Algorithms in Search, Optimization, and Machine Learning, Addison-Wesley Publishing Co., 1989.

8. Heudin, J.-C., Virtual Worlds - Synthetic Universes, Digital Life, and Complexity. Perseus Books, Reading (MA), 1998.

9. Komosinski, M., Ulatowski, Sz., Framsticks: towards a simulation of a nature-like world, creatures and evolution. In: Proceedings of 5th European Conference on Artificial Life (ECAL99), September 13-17, 1999, Lausanne, Switzerland, Springer-Verlag, 261-265.

10. Komosinski, M., Rotaru-Varga, A., From Directed to Open-Ended Evolution in a Complex Simulation Model. In: Proceedings of $7^{\text {th }}$ International Conference on Artificial Life, Portland, USA, August 2000, MIT Press.

11. Komosinski, M., Ulatowski, Sz., Framsticks Internet site, http://www.frams.alife.pl/

12. Langton, Ch., Journal of Artificial Life, Volume 1, Number 1/2, MIT Press, Fall 1993/Winter 1994.

13. Maes, P., Darrel, T., Blumberg, B., Pentland, A., The ALIVE system: full-body interaction with autonomous agents. In: Proc. of the Computer Animation '95 Conference, Geneva, Switzerland, IEEE-Press, 1995. 
14. Maes, P., Artificial Life meets Entertainment: Interacting with Lifelike Autonomous Agents. In: Special Issue on New Horizons of Commercial and Industrial AI 38, 11 (1995) 108-114, Communications of the ACM, ACM Press.

15. Michalewicz, Z.: Genetic Algorithms + Data Structures $=$ Evolution Programs, SpringerVerlag, 1996.

16. Ray, T. S., An approach to the synthesis of life, Artificial Life II, Langton C. G. et al. (eds.), Addison-Wesley, 1992, 371-401.

17. Ray, T. S., An evolutionary approach to synthetic biology: Zen and the art of creating life. In: Artificial Life 1, 1994, 195-226.

18. Reynolds, C., Flocks, herds and schools: a distributed behavioral model. In: Computer Graphics: Proceedings of SIGGRAPH '87, 21 (4), ACM Press, 1987.

19. Ronald, E. M. A., Sipper, M., Capcarrère, M. S., Testing for Emergence in Artificial Life. In: Proceedings of 5th European Conference on Artificial Life (ECAL99), September 1317, 1999, Lausanne, Switzerland, Springer-Verlag, 13-20.

20. Sims, K., Evolving 3D Morphology and Behaviour by Competition. In: R. Brooks, P. Maes (eds.), Artificial Life IV Proceedings, MIT Press, 1994, 28-39.

21. Terzopoulos, D. et al., Artificial fishes with autonomous locomotion, perception, behavior and learning, in a physical world. In: Proc. of the Artificial Life IV Workshop, Pattie Maes and Rod Brooks (eds.), MIT Press, 1994.

22. Wachowski, A., Wachowski, L., The Matrix, USA, 1999, Warner Bros, 135 min. 